Supporting Information for

\title{
Mesoporous Silica Nanospheres as Highly Efficient MRI Contrast Agents
}

\author{
Kathryn M. L. Taylor, ${ }^{\dagger}$ Jason S. Kim, ${ }^{\dagger}$ William J. Rieter, ${ }^{\dagger}$ Hongyu An, ${ }^{\ddagger}$ Weili Lin,,${ }^{\ddagger}$ and \\ Wenbin Lin*广 \\ ${ }^{\dagger}$ Department of Chemistry and ${ }^{\ddagger}$ Department of Radiology, University of North Carolina, \\ Chapel Hill, NC 27599, wlin@unc.edu
}

1. Materials and Methods. Cetyltrimethylammonium bromide (CTAB), $\mathrm{GdCl}_{3}{ }^{\circ} 6 \mathrm{H}_{2} \mathrm{O}$, bromoacetic acid, and tetraethyl orthosilicate (TEOS) were purchased from Aldrich and used without further purification. 3-(trimethoxysilylpropyl)diethylene triamine was purchased from Gelest. Thermogravimetric analysis (TGA) was performed using a Shimadzu TGA-50 equipped with a platinum pan and a heating rate of $3{ }^{\circ} \mathrm{C}$ per minute, under air. Powder X-ray diffraction (PXRD) patterns were collected on a Bruker SMART APEX II diffractometer using $\mathrm{Cu}$ radiation. The PXRD patterns were processed with the APEX 2 package using phase ID plugin. A Hitachi 4700 field emission scanning electron microscope (SEM) and a JEM 100CX-II transmission electron microscope (TEM) were used to determine particle size and morphology. A Cressington 108 Auto Sputter Coater equipped with a Au/Pd (80/20) target and an MTM-10 thickness monitor was used to coat the samples with a $5 \mathrm{~nm}$ thick conductive layer before taking SEM images. Each SEM sample was prepared by suspending the nanoparticles in ethanol. A drop of the suspension was then placed on a glass slide and the solvent was allowed to evaporate. TEM samples were also prepared from ethanolic particle dispersions on amorphous carbon coated copper grids. An Applied Research Laboratories (ARL) SpectraSpan 7 DCP spectrometer was used to measure $\mathrm{Gd}^{3+}$ concentration.

MR images were acquired on a Siemens $3 \mathrm{~T}$ Allegra (Siemens Medical Systems, Erlangen, Germany) with a CP head coil, and a 9.4 T Bruker BioSpec (Bruker Biospin, Ettlingen, Germany) system with a $35 \mathrm{~mm}$ quadrature RF transmit and receive coil. For the $3 \mathrm{~T}$ scanner, a $3 \mathrm{D}$ FLASH sequence was utilized to compute $\mathrm{T}_{1}$ maps with seven different flip angles $(2,5,10,20,30,40$, and 60). Imaging parameters were: $F O V=190 x$ $190 * 64 \mathrm{~mm}^{3}$, Matrix size $=128 \times 128 * 32, \mathrm{TR} / \mathrm{TE}=40 / 1.64 \mathrm{~ms}$, total data acquisition time was 19 minutes. A 2-D multiple echo spin echo sequence was used to estimate $\mathrm{T}_{2}$ maps. In total, 32 echoes with an echo spacing of $6.2 \mathrm{~ms}$ were obtained. The first echo time was $6.2 \mathrm{~ms}$. TR was $3000 \mathrm{~ms}$. FOV and matrix size were set to $190 \mathrm{x} 190 \mathrm{~mm}^{2}$ and $128 \mathrm{x}$ 128. The slice thickness was $2 \mathrm{~mm}$. The total data acquisition time was about 6 minutes and 29 seconds. All images were acquired on a 9.4 T Bruker BioSpec (Bruker Biospin, Ettlingen, Germany) system with a $35 \mathrm{~mm}$ quadrature RF transmit and receive coil. For the 9.4 $\mathrm{T}$ scanner, two $2 \mathrm{D}$ multiple echo spin echo sequences were used to estimate $\mathrm{T}_{2}$ maps for a wide range of $T_{2}$. FOV and matrix size were set to $35 \times 30 \mathrm{~mm}^{2}$ and $128 \mathrm{x}$ 128. The slice thickness was $1 \mathrm{~mm}$. In total, 32 echoes with an echo spacing of $3.7 \mathrm{~ms}$ for sequence 1 and 40 echoes with an echo spacing of $10 \mathrm{~ms}$ for sequence 2 were obtained. The TR/TE1 were $3.7 / 3000 \mathrm{~ms}$ and $10 / 4114 \mathrm{~ms}$ for each sequence, respectively. The total data acquisition time was about 10 minutes. $\mathrm{T}_{1}$ maps were 
obtained with a variable TR rapid acquisition relaxation enhanced (RARE) sequence with a TE $=5.14 \mathrm{~ms}$. Twenty two variable TRs $(\mathrm{TR}=18,25,40,60,90,120,160,200,250$, $300,380,500,700,1000,1500,2000,2500,3000,4000,5000,6000,7000 \mathrm{~ms})$ were used with a FOV of $35 \times 30 \mathrm{~mm}^{2}$ and a matrix size of $128 \times 128$. RARE factor was 3 and total data acquisition was about 24 minutes.

\section{Ligand and Gd-Complex Synthesis.}

3-Aminopropyl(trimethoxysilyl)diethylenetriamine tetraacetic acid (SiDTTA) and its Gd complex. The Si-DTTA ligand and Si-DTTA-Gd complex were synthesized as described previously. ${ }^{1}$

\section{Synthesis of Mesoporous Silica Nanoparticles}

MSN. $0.100 \mathrm{~g}(0.274 \mathrm{mmol})$ of cetyltrimethylammonium bromide (CTAB) was dissolved in $48 \mathrm{~mL}$ of distilled $\mathrm{H}_{2} \mathrm{O}$, along with $0.35 \mathrm{~mL}$ of $2 \mathrm{M}$ sodium hydroxide. This solution was then heated to $80^{\circ} \mathrm{C}$. Once the reaction had reached $80^{\circ} \mathrm{C}, 0.50 \mathrm{~mL}(0.47 \mathrm{~g}$, $2.24 \mathrm{mmol}$ ) of tetraethylorthosilicate (TEOS) was rapidly injected. The reaction was then stirred for an additional 2 hours at $80^{\circ} \mathrm{C}$. At the completion of the reaction the particles were isolated by centrifuging at $10,000 \mathrm{rpm}$ for 10 minutes. They were then washed with $\mathrm{H}_{2} \mathrm{O}$ and ethanol, before being redispersed in $30 \mathrm{~mL}$ of ethanol. Yield: $171 \mathrm{mg}$. The particles obtained are approximately $75 \mathrm{~nm}$ in diameter.

Extraction. The surfactant was extracted from the mesoporous materials using a $1 \mathrm{wt} \%$ solution of sodium chloride in methanol. The particles were dispersed in the $\mathrm{NaCl} / \mathrm{MeOH}$ solution at a concentration of approximately $3.5 \mathrm{mg} / \mathrm{mL}$. After stirring at room temperature for 3 hours, the particles were isolated by centrifuging, and then redispersed in another aliquot of the $\mathrm{NaCl} / \mathrm{MeOH}$ solution. The extraction was performed for a total of three times. After the final extraction, the particles were washed with distilled $\mathrm{H}_{2} \mathrm{O}$ and ethanol, before being redispersed in ethanol.

\section{Coating of Mesoporous Silica Nanoparticles with Si-DTTA-Gd complex}

MSN-Gd. Approximately $160 \mathrm{mg}$ of extracted MSN particles were suspended in $10 \mathrm{~mL}$ of toluene. $0.78 \mathrm{~mL}(0.075 \mathrm{mmol})$ of a $0.097 \mathrm{M}$ solution of a Si-DTTA-Gd aqueous solution was then added. The mixture was then heated to reflux overnight. The particles were isolated by centrifuging, and washed with distilled water and ethanol, before being redispersed in ethanol. Yield: $132 \mathrm{mg}$. In order to ensure there was no excess Gd remaining on the silica, the particles were washed twice with a $\mathrm{pH}=3$ solution by sonicating for 10 minutes. They were then washed once with distilled water before being redispersed. The particles were also dialyzed against distilled water for 6 hours, changing the water once an hour. TGA analysis showed an initial weight loss of $13.7 \%$ from room temperature to $130{ }^{\circ} \mathrm{C}$ for the adsorbed solvent species and a further weight loss of $10.5 \%$ from $225-500{ }^{\circ} \mathrm{C}$ for the organic components of Si-DTTA-Gd. 


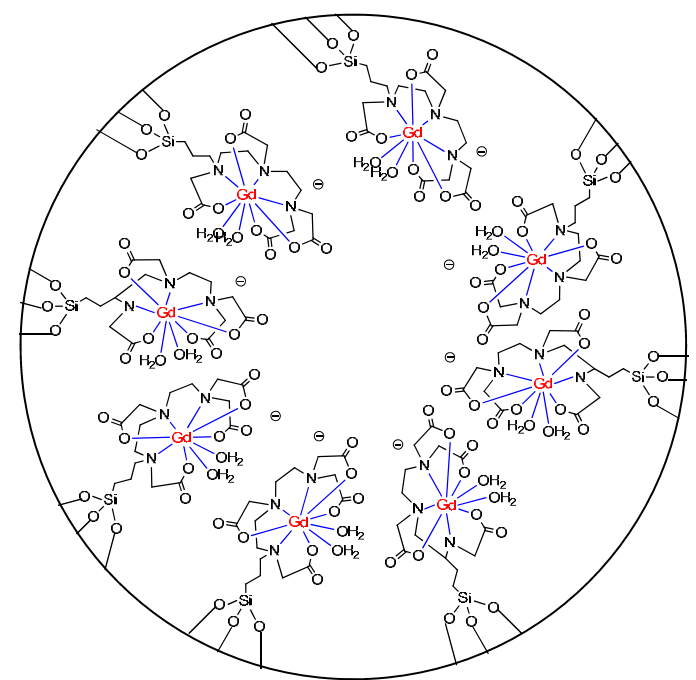

Figure S1. Schematic presentation of the Gd-Si-DTTA chelates that have been grafted onto the interior walls of MSN nanoparticle via the siloxane linkage.

\section{Fluorescent MSN Particles}

Synthesis of Rhodamine-APS. $6.8 \mathrm{mg}(0.0127 \mathrm{mmol})$ of rhodamine B isothiocyanante was dissolved in $1.1 \mathrm{~mL}$ of ethanol. $3.3 \mu \mathrm{L}(3.1 \mathrm{mg}, 0.0141 \mathrm{mmol})$ of 3 aminopropyltriethoxy silane was then added, and the reaction was stirred at room temperature, under $\mathrm{N}_{2}$, and in the dark for 24 hours. At the completion of the reaction the solution was diluted to a total volume of $2 \mathrm{~mL}$ with additional ethanol to make a solution with a rhodamine-APS concentration of approximately $6 \mathrm{mM}$.

MSN-Gd-1. Approximately $50 \mathrm{mg}$ of extracted MSN particles were suspended in $4 \mathrm{~mL}$ of toluene. $170 \mu \mathrm{L}(0.016 \mathrm{mmol})$ of a $0.097 \mathrm{M}$ solution of a Si-DTTA-Gd aqueous solution and $110 \mu \mathrm{L}(0.00066 \mathrm{mmol})$ of a $6 \mathrm{mM}$ solution of rhodamine-APS in ethanol were then added. The mixture was then heated to reflux overnight. The particles were isolated by centrifuging, and washed with distilled water and ethanol, before being redispersed in ethanol. Yield: $48 \mathrm{mg}$. In order to ensure there was no excess $\mathrm{Gd}$ remaining on the silica, the particles were washed twice with a $\mathrm{pH}=3$ solution by sonicating for 10 minutes. They were then washed once with distilled water before being redispersed.

\section{Characterization of Materials}



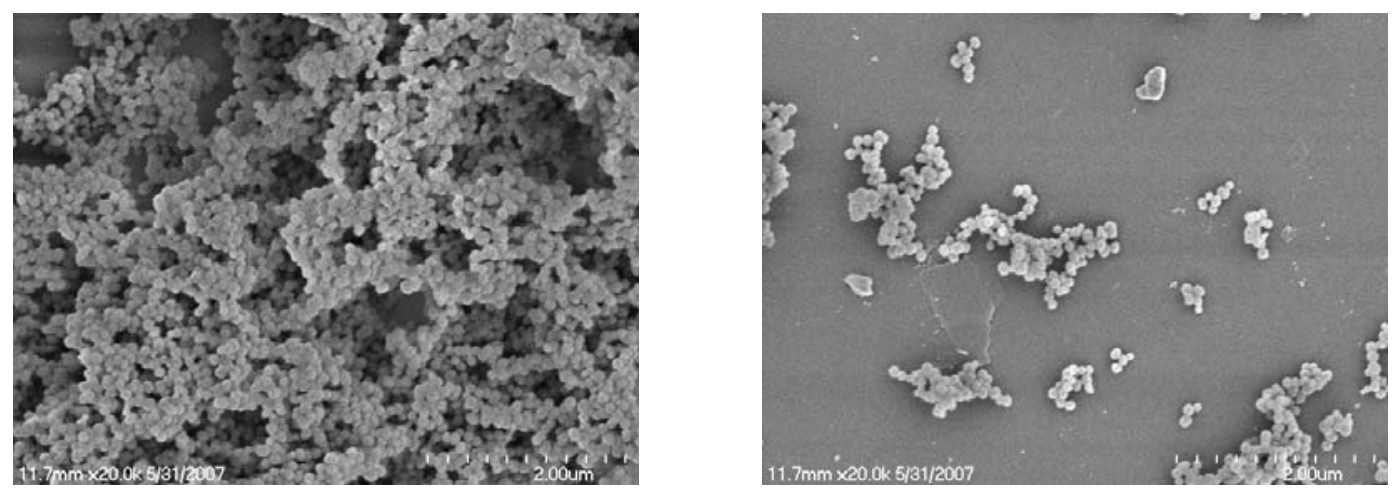

Figure S2. SEM images of surfactant-extracted MSN particles.
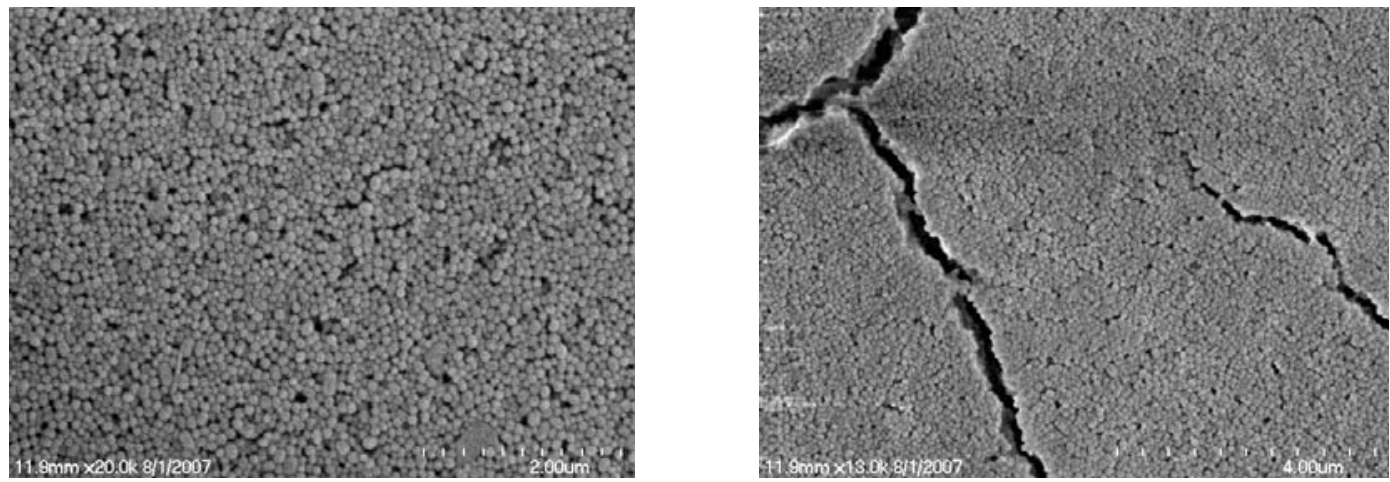

Figure S3. SEM images of MSN-Gd particles.
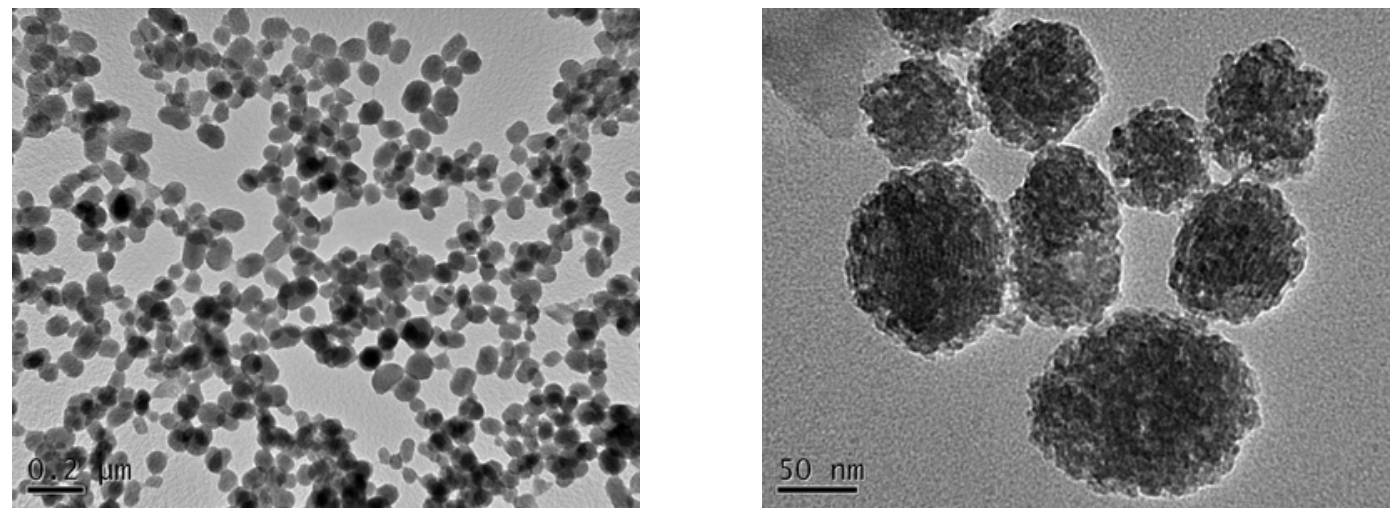

Figure S4. TEM images of surfactant-extracted MSN particles. 

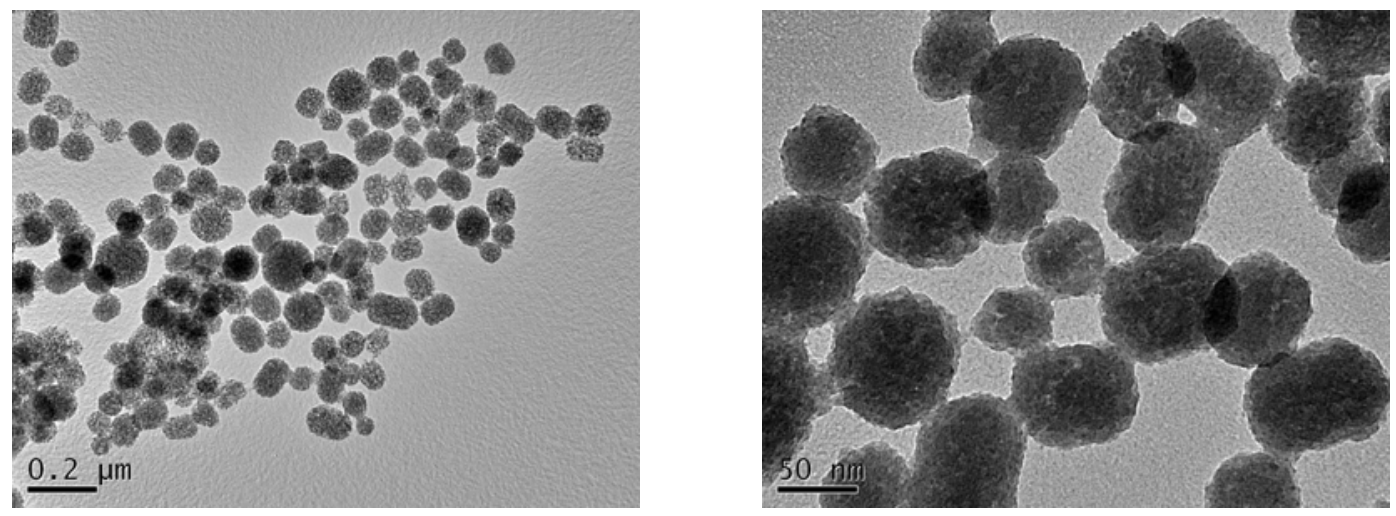

Figure S5. TEM images of MSN-Gd particles.

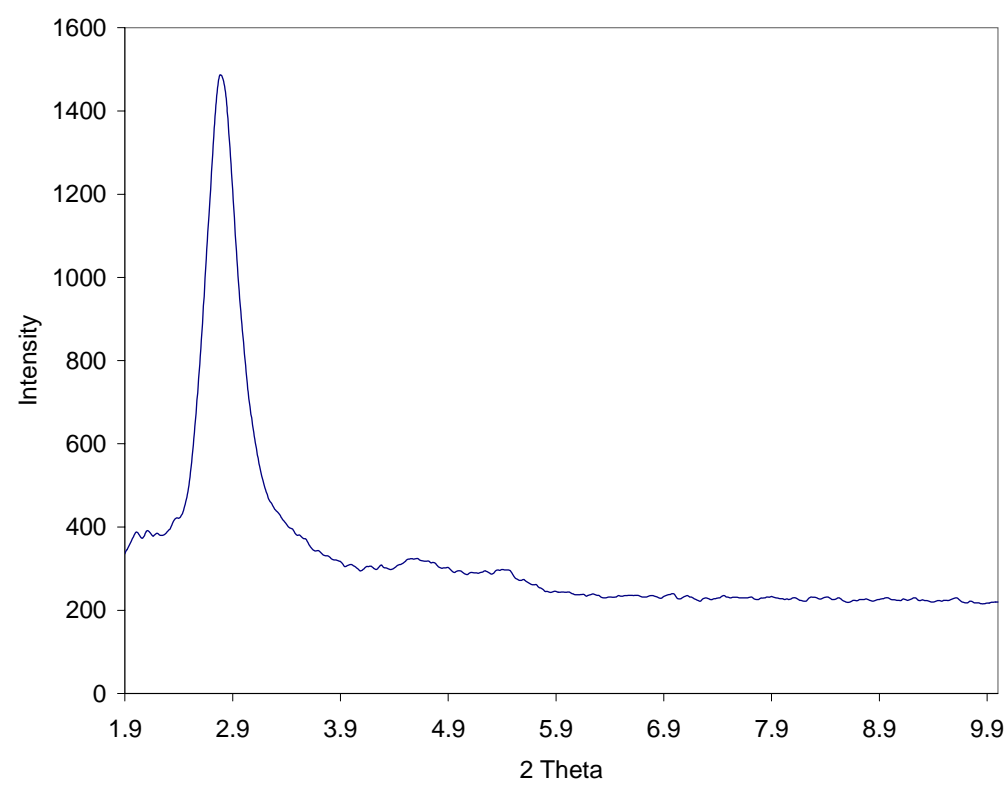

Figure S6. Powder X-ray diffraction pattern of MSN, showing peaks at $2.8^{\circ}, 4.6^{\circ}$, and $5.4^{\circ}$. 


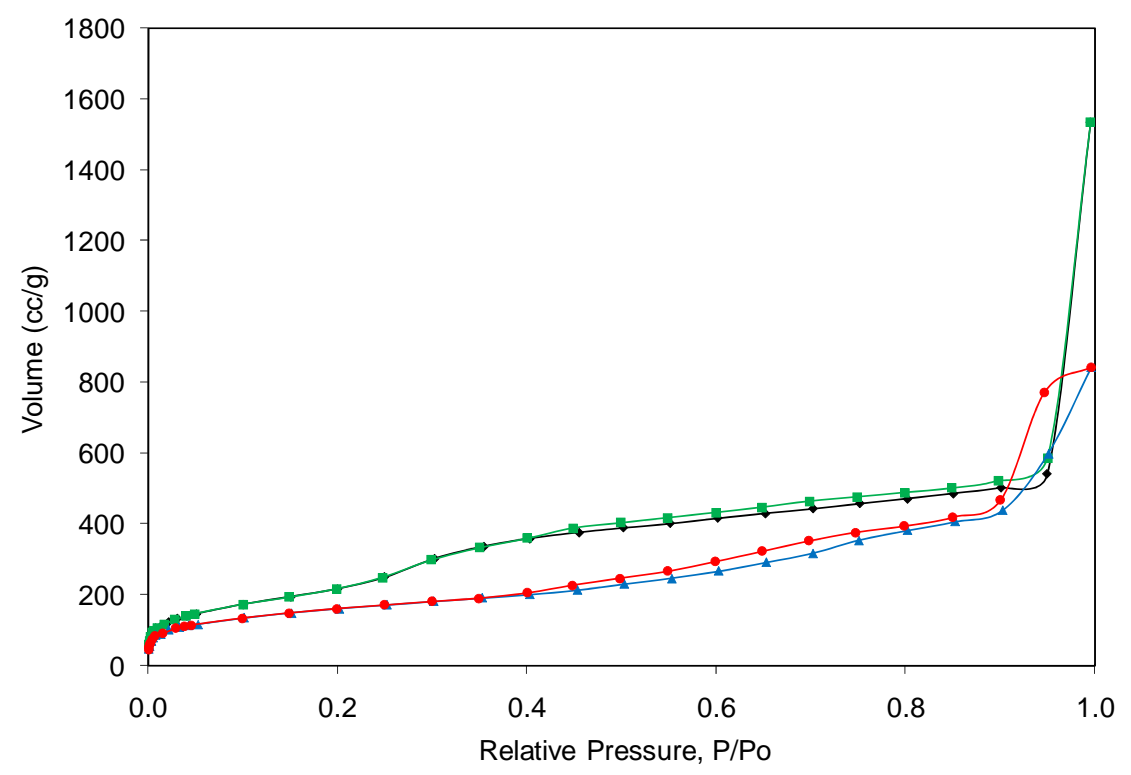

Figure S7. Nitrogen adsorption isotherm of surfactant-extracted MSN (black-adsorption; green-desorption) and MSN-Gd (blue-adsorption; red-desorption).

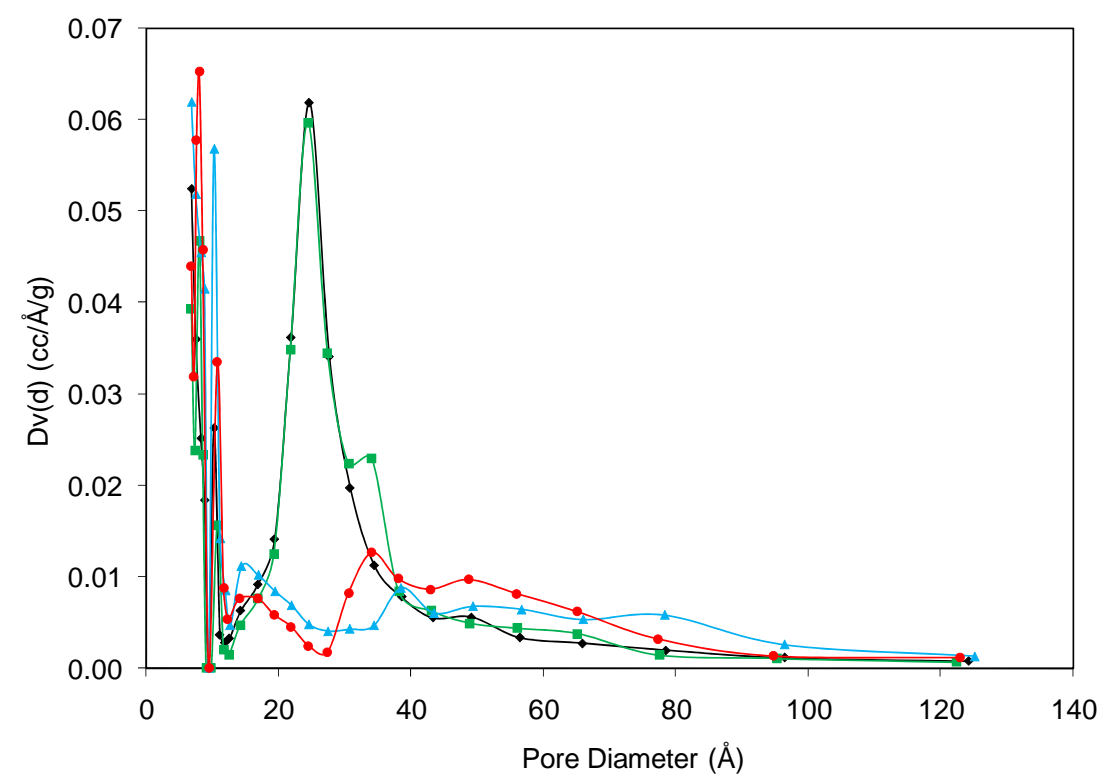

Figure S8. Pore size distribution of surfactant-extracted MSN (black-adsorption; greendesorption) and MSN-Gd (blue-adsorption; red-desorption).

\section{Biological Assays}

Murine monocyte cell culture. Cells (ATCC\# TIB67) were purchased from the UNC Lineberger Comprehensive Cancer Center's Tissue Culture Facility. Cells were maintained in DMEM-H containing 10\% FBS and 1\% penicillin/streptomycin at $37^{\circ} \mathrm{C}$ and $5 \% \mathrm{CO}_{2}$. 
MTS cell viability assay. Manufacturer's (Promega) protocol was used to determine cell viability of monocytes in the presence of MSN-Gd nanoparticles. Briefly, monocytes are placed in a 96-well plate at a concentration of 5000 cells $(100 \mu \mathrm{L})$ per well and various amounts of particles are added in a $20 \mu \mathrm{L}$ volume of distilled $\mathrm{H}_{2} \mathrm{O}$. Each particle concentration is replicated seven times, and $100 \%$ viability is standardized to $20 \mu \mathrm{L}$ distilled $\mathrm{H}_{2} \mathrm{O}$ without nanoparticles. The cells are incubated with nanoparticles for 22 hours before addition of MTS. An additional 4 hours for MTS metabolism is allowed before reading plate absorbance at $492 \mathrm{~nm}$. Wells with nanoparticles and without cells are used for background correction.

In vitro labeling of murine monocytes. Cells are detached from resident flasks using a cell scraper and re-plated into 6-well plates at a concentration of 300,000 cells per well. Monocyte containing plates are maintained at $37^{\circ} \mathrm{C}$ and $5 \% \mathrm{CO}_{2}$ for 24 hours, at which point $4 \mu \mathrm{g}$ nanoparticles per well ( $2 \mathrm{~mL}$ media) are added. Cells are allowed to incubate with nanoparticles at $37^{\circ} \mathrm{C}$ and $5 \% \mathrm{CO}_{2}$ for 30 minutes before extensive washing. The resulting labeled cells are imaged using an Olympus FV500 Confocal Laser Microscope at 60X using a RITC filter and DIC settings.

\section{In vivo animal imaging studies}

$\mathbf{T}_{2}$-weighted midbody MR imaging. A 6-week old male DBA/1J mouse is imaged in a Bruker 9.4T Small Animal scanner. After pre-contrast image acquisition of animal midbody, $2.5 \mathrm{mg}$ of MSN-Gd NPs are intravenously injected via tail vein. This corresponds to a dose of $31 \mu \mathrm{mol} / \mathrm{kg}$. The animal is imaged at time points of 30 minutes, 1 day, 2 day, 4 day, and 17 day post-contrast administration.

$\mathrm{T}_{1}$-weighted midbody MR imaging and relaxation time mapping. A 6-week old female DBA/1J mouse was imaged in a Bruker 9.4T Small Animal scanner. After pre-contrast image acquisition of animal midbody, an appropriate dose of MSN-Gd NPs was intravenously injected via tail vein. The animal was imaged at time points of 15 minutes, 1 hour, 24 hours post-contrast administration.

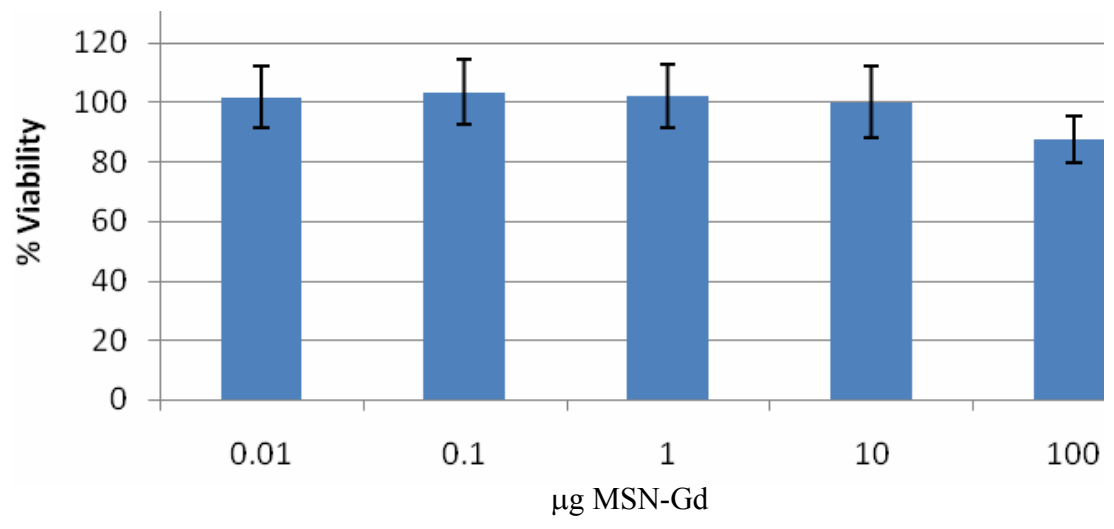

Figure S9. MTS assay for monocyte cell viability after incubation with different amounts of MSN-Gd NPs. 


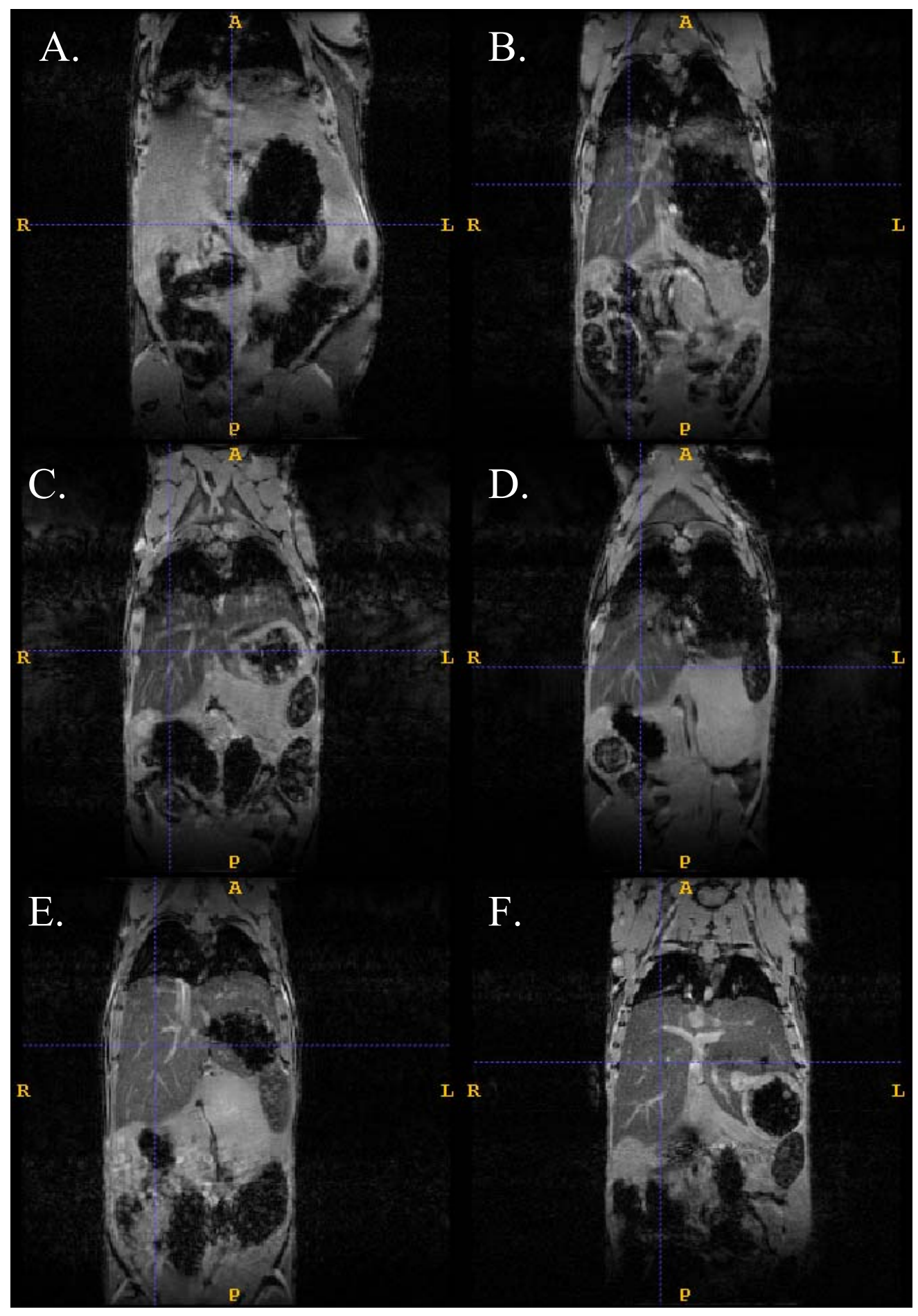

Figure S10. Midbody images focused on liver of (A) pre-contrast, (B) 30 minutes, (C) 1 day, (D) 2 day, (E) 4 day, and (F) 17 day post contrast administration via intravenous injection of a $31 \mu \mathrm{mol} / \mathrm{kg}$ dose of MSN-Gd. 


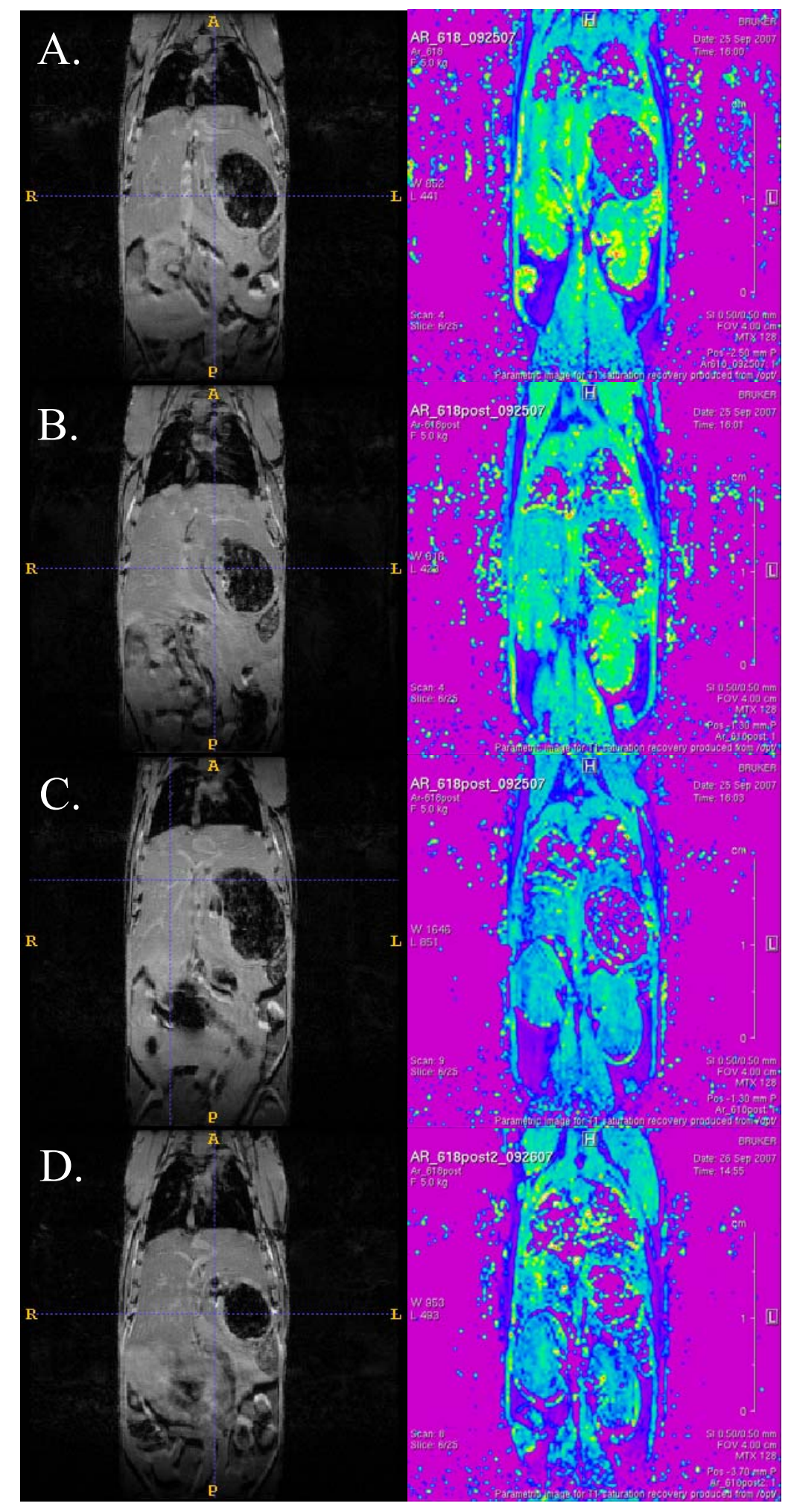

Figure S11. MR imaging and the associated $\mathrm{T}_{1}$ relaxation time mapping (A) pre-contrast injection, (B) 15 minutes, (C) 1 hour, and (D) 24 hour post-constrast administration via intravenous injection of a $4.15 \mu \mathrm{mol} / \mathrm{kg}$ dose of MSN-Gd. 


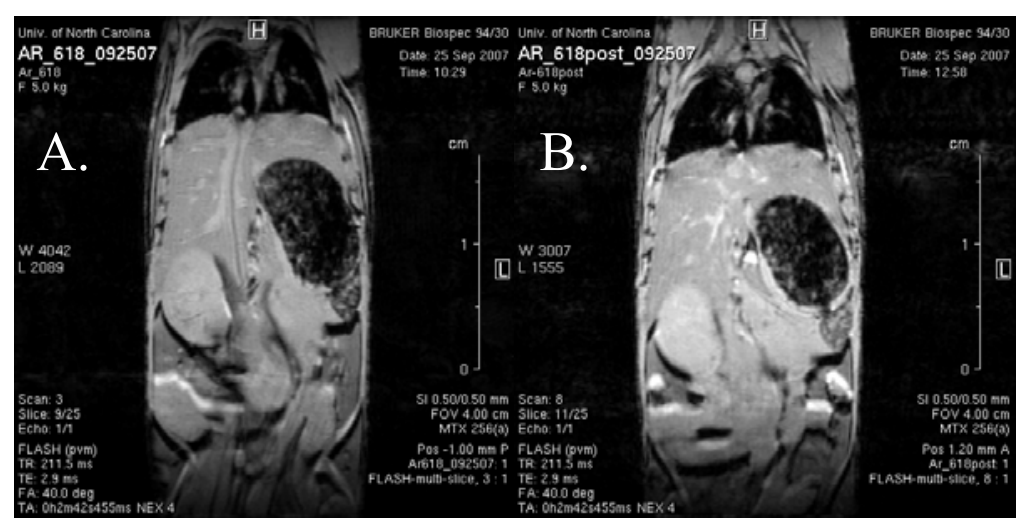

Figure S12. MR images (A) pre-contrast and (B) 1 hour post-contrast $(4.15 \mu \mathrm{mol} / \mathrm{kg}$ dose) after rescaling using data from $\mathrm{T}_{1}$ relaxation time maps. This result shows the ability of MSN-Gd in enhancing the $\mathrm{T}_{1}$-weighted liver images at lower doses.
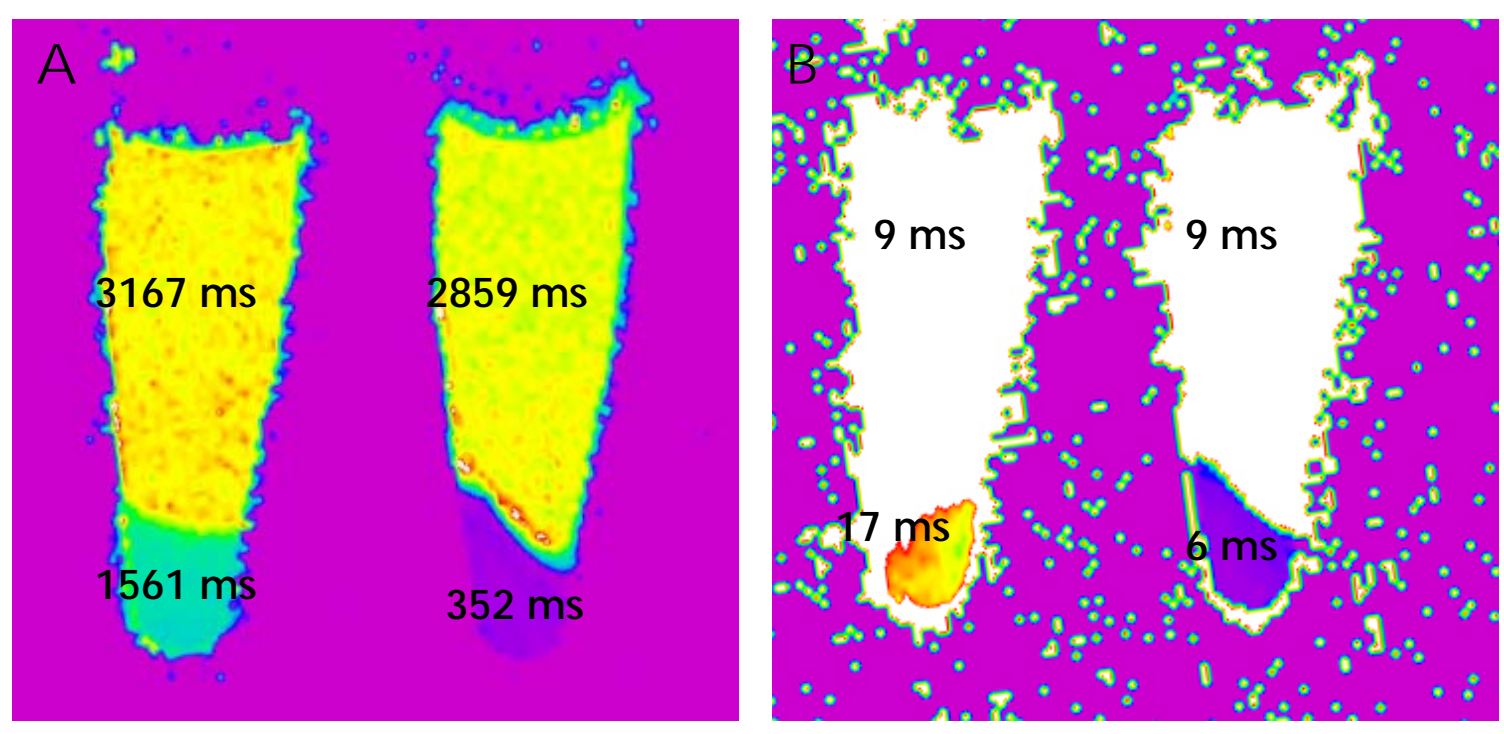

Figure S13. (A) $\mathrm{T}_{1}$-Relaxation time maps for monocyte cell pellets incubated without MSN-Gd (left) and with $0.3 \mathrm{mg} \mathrm{MSN-Gd}$ for $5 \times 10^{6}$ cells in $3 \mathrm{~mL}$ of media (right). (B) $\mathrm{T}_{2}$-Relaxation time maps for monocyte cell pellets incubated without MSN-Gd (left) and with $0.3 \mathrm{mg}$ MSN-Gd for $5 \times 10^{6}$ cells in $3 \mathrm{~mL}$ of media (right) 
a)

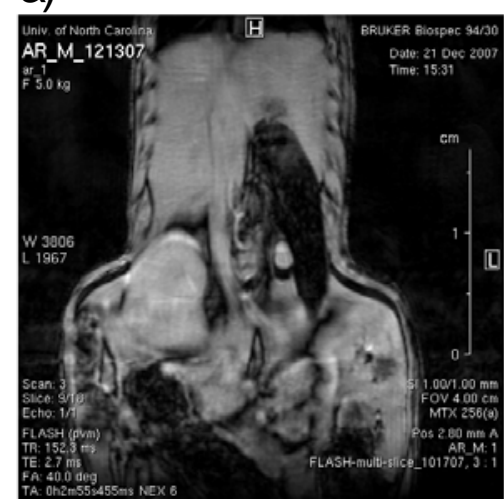

b)

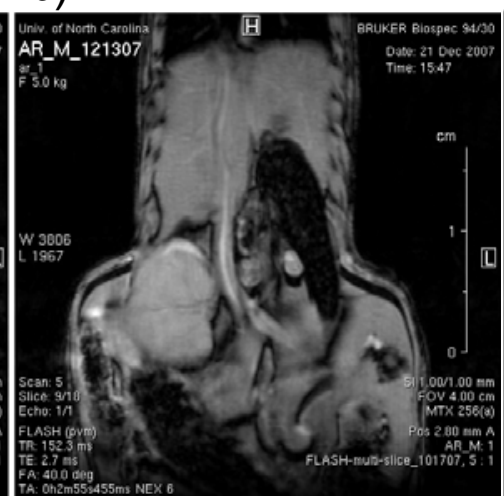

c)

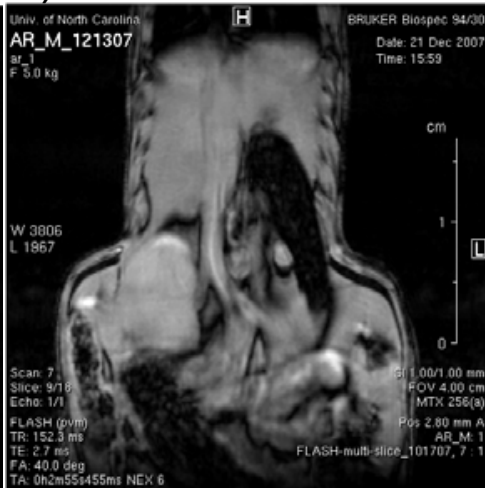

Figure S14. (a) Pre-contrast, (b) 16 minutes post-contrast, and (c) 28 minutes postcontrast $T_{1}$-weighted mouse MR image following tail vein injection of $2.1 \mu \mathrm{mol} / \mathrm{kg}$ dose of Gd-Si-DTTA complex. Only slight T1-weighted aorta signal enhancement is seen (as compared to MSN-Gd).

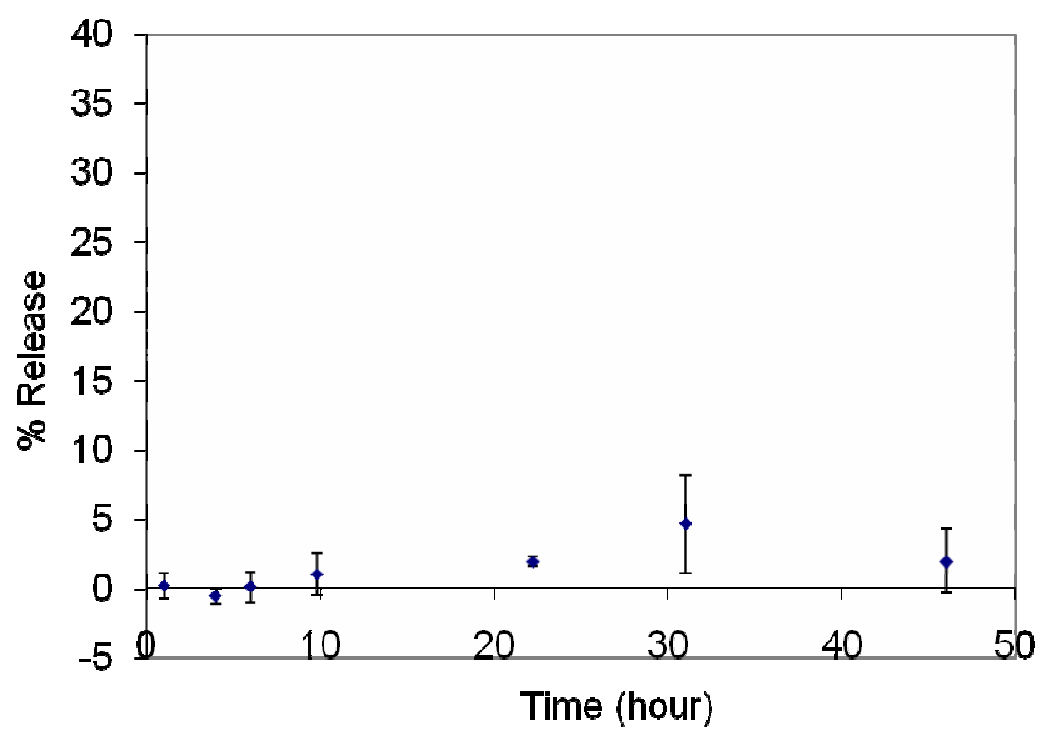

Figure S15. Percentage of Gd-containing species that have been released from the MSN$\mathrm{Gd}$ materials in water at $37.4{ }^{\circ} \mathrm{C}$ as a function of time. This result shows that the Gdcontaining species have been completely removed from the MSN materials during the workup and the Gd-Si-DTTA chelate are stable under physiologically relevant conditions.

\footnotetext{
${ }^{1}$ Rieter, W.R.; Kim, J.; Taylor, K.M.L.; An, H.; Lin, W.; Tarrant, T.; and Lin, W. Angew. Chem. Int. Ed. 2007, 46, 3680-36982.
} 